\title{
A Profound Insight of Cardiopulmonary Resuscitation (CPR) in Pakistan
}

\author{
Asif Siddiqui*1,2, Nameera Ahmed ${ }^{2}$, Aaisha Ahmed ${ }^{2}$, Annum Aslam² \\ ${ }^{1}$ Dow University of Health Sciences, Karachi, Pakistan. \\ ${ }^{2}$ BoschMedicus, Karachi, Pakistan.
}

\section{INTRODUCTION}

Sudden Cardiac Arrest is considered as a major unresolved health issue and prevalence of cardiac emergencies are common in Pakistan. Many people are suffering from different cardiac diseases which include heart attack, cardiac arrest and congestive heart failure. Multiple deaths are reported especially in rural areas due to sudden cardiac events.

Cardiac arrest, also known as cardio-pulmonary arrest or circulatory arrest, occurs suddenly causing complete cessation of cardiac activity and is confirmed by loss of effective circulation [1], as a result the blood flow towards the brain progressively worsen which further causes difficulty in breathing. This combined effect of depression in breathing and loss of effective circulation leads to ischemia and causes sudden death [2].

Early minutes are very crucial to increase survival rate because in cardiac arrest, time is key.

Once the normal systemic circulation stops, all vital organs of the body are poorly perfused. As a result of this decreased blood flow which causes the brain to get more quickly and severely affected.

The brain is most sensitive to hypoxia than other body organs. Without oxygen, neurons can begin to die in about 4 to 6 minutes, and this damage can become permanent and irreversible after about 10 minutes. Death can occur if the person does not receive immediate emergency care.

The patient becomes unconscious and breathing becomes difficult or absent, if this condition of cardiac arrest persists, permanent brain damage may occur and pulsation of carotid pulse diminishes [3].

Cardio-Pulmonary Resuscitation is a life saving effort that combines external chest compression and artificial ventilation, to make the heart pump and assisted breathing for the victim. CPR expands the window of time until the emergency medical help arrives [1].

It keeps the blood to circulate in the body to sustain a victim

*Address correspondence to this author at the Dow University of Health Sciences and BoschMedicus, Karachi, Pakistan. Email: asif.aha82@gmail.com until the trained and well-equipped help arrives on the scene to restore the return of spontaneous circulation.

CPR is the most important life-saving skill practiced throughout the world because every day, there are many events that can result in serious injury and cause a person to collapse suddenly. These occurrences require fast action and those people who have knowledge and are capable enough to provide helpful assistance.

If CPR and defibrillation are delayed, the survival chances would be reduced by $10 \%$ [3].

Automated External Defibrillator, is a portable device that automatically detects the life threatening abnormal cardiac rhythms and are used to give external shock to cardiac muscles to restore their normal contractions and allows heart to resume beating at its normal pace [2]. The only definitive medical way for treating Ventricular Fibrillation (VF) is defibrillation. The early help of a victim with effective CPR and defibrillation has been shown to increase the chances of survival in cardiac arrest [3].

A survey based study was conducted on students in Jinnah Medical \& Dental College (JMDC), Dow International Medical College (DIMC) which was based on the response of students regarding CPR. The study showed that the medical students had knowledge about CPR but they had poor motor skills because most of the students didn't attend CPR or BLS class [4].

\section{DISCUSSION}

More than 700 medical professionals from different departments including Consultant, Professor, Assistant Professor, CMO's, RMO's, MO's, HO's, PG's and GP's were trained at BoschMedicus, that is affiliated with Dow University of Health Sciences (DUHS) and DUHS is an International Training Centre of American Heart Association (AHA). Around 3000 non-medical professionals were given the awareness of CPR in major cities and peripheries of Pakistan. In Pakistan, we observed that the medical professionals had awareness and knowledge regarding CPR but due to less implementation of their motor skills, they are not upto those standards as recommended by American Heart 
Association (AHA). Non-medical professionals were aware of CPR but they were lacking the knowledge and skills of CPR.

\section{CONCLUSION}

Cardiovascular emergencies occurs within a blink of an eye and are very threatening events to life that must be recognized as early as possible and treated immediately on time to avoid delay in help which increases the survival of the victim. To conclude, medical professional had knowledge about CPR but they have poor motor skills whereas non-medical professionals and lay rescuers neither had knowledge nor the skills to perform CPR. Therefore, it is very important that every person in the community should know about the CPR skills to save lives and improve the survival rate. Along with medical and non-medical professionals we must aware the lay person regarding CPR, because sudden cardiac arrest mostly occurs at home therefore it's our responsibility to spread awareness among community. So that they must be able to recognize potential cardiac events and be prepared to provide resuscitative efforts at any time.

\section{CONFLICT OF INTEREST}

Declared None.

\section{ACKNOWLEDGEMENTS}

Declared None.

\section{REFERENCES}

[1] American Heart Association, European Resuscitation Council, Australian Resuscitation Council, et al. Cardiac arrest and cardiopulmonary resuscitation outcome reports. Circulation 2004; 110: 3385-97.

DOI: 10.1161/01. CIR.0000147236.85306.15.

[2] Zamir Q, Nadeem A, Rizvi AH. Awareness of cardiopulmonary resuscitation in medical-students and doctors in Rawalpindi-Islamabad, Pakistan. J Pak Med Assoc 2012; 62(12): 1361.

[3] American Red Cross. First AID/CPR/AED participant's manual. 2018; Available from: http://www.mtech.edu/env_health_safety/emergency/docs/facpr_participant_manual.pdf

[4] Zaheer H, Haque Z. Awareness about BLS (CPR) among medical students: Status and requirements. J Pak Med Assoc 2009; 59(1): 57-9. 